\title{
Embedded Research in the Design of Interactive Technologies for Children with Special Educational Needs: An Account of Research in Progress
}

\author{
Keir Williams \\ Queen Mary University of London \\ London \\ keir@eecs.qmul.ac.uk
}

\author{
David Meckin \\ Queen Mary University of London \\ London \\ dcm@eecs.qmul.ac.uk
}

\author{
Tony Stockman \\ Queen Mary University of London \\ London \\ tony.stockman@eecs.qmul.ac.uk
}

\author{
Nick Bryan-Kinns \\ Queen Mary University of London \\ London \\ nick.bryan-kinns@eecs.qmul.ac.uk
}

\begin{abstract}
Conducting longitudinal interaction design research within an institution that caters for young people with special educational needs (SEN) is a challenging but fascinating task. This paper aims to elucidate some of the key factors that have emerged from conducting such research with students and staff in Three Ways School in Bath, UK. The various institutions and people involved are detailed as well as the most salient points for consideration when undertaking studies in the SEN context. The points are then reflected upon drawing on concrete examples from the researchers' own experiences. These experiences, both positive and negative, may be of use to other researchers and practitioners when conducting research studies in the SEN classroom context and other potentially sensitive contexts.
\end{abstract}

Designing Interactive Systems for Children, Special Educational Needs, Research Methods.

\section{INTRODUCTION}

Novel interactive technologies have the potential to help young people with special educational needs (SEN) express themselves creatively and develop new understandings about their own actions and the world around them. The authors believe it is imperative that rigorous, ethical and reflexive methods be employed when carrying out research into the design and evaluation of such technologies. Undertaking design research in this field is a complex, yet rewarding practice, which can present many unforeseen issues. The purpose of this paper is to outline and reflect upon the experiences of working in the setting of a SEN classroom in the UK. In detailing the main factors that have been encountered thus far, it is intended that other research students and early-career researchers, as well as practitioners, could find this information useful when conducting their own future studies.

The first section briefly sets out the aims of the studies and the approach taken. The ensuing section details the stakeholders who have active roles within the two research projects. This will help elucidate the institutional setting within which the research is situated.

1 The first two authors of this paper are studying at Queen Mary

University, London as part of the Media and Art Technologies doctoral training program. They are also both currently

conducting individual research projects in partnership with Three

Ways School in Bath, UK. Three Ways School is a special

needs school that supports around 150 children and young

adults with a broad range of sensory and educational needs.
The authors outline the key factors in the process of carrying out research in the school. Using examples from the researchers' own experiences, they reflect upon the main points to provide greater insight into both the positive and negative aspects of the studies. Finally, the conclusions and further considerations are discussed.

A number of approaches have been proposed and undertaken for conducting design research with special needs students in schools and social settings. They offer a range of best practices, concerns and practical examples for researching in this rewarding field $[2,3,4$, $5,6,7,8,9,10]$. While this document is not presented as a set of best practices, the authors believe that the details outlined may be of use to other researchers and practitioners undertaking projects designing interactive technologies for young people with special educational needs and for researchers conducting projects in other sensitive contexts.

\section{AIMS}

One of the common themes running through both researchers interests is the importance placed on spending extended periods of time embedded in the school in order to gain a deeper understanding of the context in which their future design interventions will be situated. The aim is that this understanding will span three levels. From the micro-level of specific students and staff, their interactions in the classroom; through the meso-level of this particular institution and its functionality; up to the macro-level of how technologies 
can be designed and used for the Special Educational Needs (SEN) classroom contexts in general.

The desired outcomes are that, having developed this understanding and forged strong relationships within the institution, the researchers will be able to design interventions that can address the gathered requirements as comprehensively and rigorously as they can. Additionally, gaining extended access to design partners may help generate more detailed and flexible frameworks for evaluation, when assessing the efficacy of any interventions that are made. Finally, conducting design research in this way could result in a lasting legacy with which the participants involved can gain more sustained use and development of the designs or frameworks beyond the initial scope of the project. This approach brings with it a number of ethical considerations that may not be present in a more controlled, experimental setting.

\section{STAKEHOLDERS}

Conducting research in a special needs school requires the researcher to negotiate with a wide range of stakeholders with differing responsibilities and sometimes competing agendas. The range of people and institutions that the researchers have needed to negotiate with during their research in special needs classrooms are discussed.

\subsection{Consider the school as an institution}

A special needs school is an institution that serves multiple roles for its staff, students and wider society. A school functions within shifting political, financial and ethical pressures that define and constrain the objectives and agendas that it works towards. Odom et al. [10] point out that the remit of a special needs school extends beyond the classroom into a much broader set of contexts, including healthcare and social services in the home, social, and vocational settings. The school with which the research is being conducted are in a position of power that they can assert in their relationship with the researcher. They ultimately have the final say whether any research is conducted there and when it is conducted if sanctioned.

\subsection{Staff}

The schools in which the authors have conducted their studies have had a wide range of staff with whom they have needed to work and negotiate with. These include head teachers, teachers, teaching assistants, therapists, access specialists, technical staff and administration staff.

\subsection{Students}

Children and young people exist within social and institutional contexts that are as complex as those inhabited by adults [1]. Within a class in a special needs school, children and young people will have a range of abilities, impairments, learning styles and social/economic backgrounds, all of which fluctuate over time. A researcher must consider this variability of participants as well as the social and institutional context in which they inhabit when considering their interactions. An ethical and methodological issue that arises from this variability in participants is that of informed consent. Participants may have impairments in speech, understanding of questions and concepts and use third parties to communicate. These elements may make gaining informed consent from participants difficult if not impossible. When research concerns participants' care provision, this can be further complicated when the subject under discussion may be the third parties that participants rely on to communicate such as communication devices, teaching assistants and parents.

\subsection{Parents/ Guardians}

Parents and guardians need to make informed decisions as to whether their child participates in a study and to what extent they themselves may contribute to the study. Much of the communication between researcher and parent is conducted at a remove through documents students take home and/ or through staff at the school. Ensuring parents and guardians are given clear and comprehensible information on which to base their informed consent is doubly important as they are not only giving consent as the guardian of a child but also as a proxy for the child.

\subsection{Researcher}

As researchers the authors wish to contribute to the school and to complement the work of its staff. At the same time, they have their own agendas and timetables for completing a successful study within the school and within a given amount of time.

\subsection{The Research Institution}

Queen Mary University of London, the research institution in the case of the authors of this paper, share the agendas to complete timely and publishable research. The university also has a responsibility to audit the ethical aspects of a study. The ethics processes can present several difficulties when pursuing an embedded approach. The ethics committee at Queen Mary sits once a month and does not sit during university holidays. When engaging in an explorative, embedded approach to research, the aims and objectives of the study and the agendas of its the people and institutions involved change over time. The length of time it takes to submit and receive ethical approval makes it more difficult to adapt studies quickly in response to these changes in context, participants and research aims.

\section{POINTS FOR CONSIDERATION}

\subsection{Contextual Factors}

Working with any groups of young people is a privileged task requiring hard work, discretion, and patience among many other skills. Particularly when working in the special educational needs classroom, researchers should always keep in mind the vulnerable and sensitive nature of the participants being studied. This may seem like a moot point to people with previous experience of working in this field, however it is nonetheless important because it requires thorough attention to the diverse and nuanced behavioral traits exhibited by the participants. When collecting information with participants, data 
protection and in particular disclosure becomes an important issue. Working within Threeways School has meant not only working within the university's but also within the schools strict data protection policies. This is a necessary aspect of working with vulnerable participants, but can restrict the way research is carried out. An example of this was that any video or images of children the researchers made could not leave the school. This made analyses of the imagery difficult and time consuming. Special Educational Needs schools in the UK are busy institutions working under pressures coming from many different angles. The authors feel very privileged to have the opportunity to work with Three Ways School as they have been welcoming from the beginning of the studies there. However, gaining initial access to potential participants in a school can be challenging as it requires a significant amount of work on the part of the school to undertake the necessary checks, and to find staff that are willing to allow researchers into their classrooms. Some research projects may also require specifically timetabled sessions in which case the process of negotiation may be more complex and sustained.

Without spending all the school hours with the students, it is very difficult to gain the depth of knowledge about them that the teachers and teaching assistants mostly already have. Consequently, it is imperative that the input of such professionals be called for and taken into account from the outset. Therefore the researchers have had to consider carefully which staff members must be included as participants in any ethical reviews.

The nature of the school environment is often that the dynamic of the setting in which the research is being carried out fluctuates greatly. This means that scheduled sessions can be postponed due to unforeseen circumstances such as students being absent or last minute timetabling changes. As the teachers are generally busy, it should be the researchers responsibility to contact the relevant parties to confirm a session is due to occur close to the time. It is also possible (as detailed below) that the session could be cancelled on the day and it is important for researchers not to become frustrated by this as it part of the nature of conducting work in this kind of environment.

\subsection{Managing Relationships}

One of the main issues when managing relationships with the various parties involved in the research project is clear communication. It is extremely important that contact is maintained throughout the many phases of the project. When a phase is entered that means a researcher may not be present at the school for some weeks, it should be clearly and effectively communicated to all the staff and students who are involved. This is not just due to common courtesy but also so that the project is kept in the mind of the participants as it can easily slip and lose priority within the myriad of other activities in the school.

While negotiating initial access can be a demanding process, ensuring that this access is maintained regularly enough is also another factor for consideration. This can be done so by keeping in contact with the staff at the school with updates on how the project is progressing.

The notion of reciprocity is another facet when conducting research in SEN schools: this relates to researchers making an effort to undertake tasks that differentiate them from being mere observers. Whether this be acting as a teaching assistant, helping with extra curricular activities or running creative projects in the school, this shows a willingness and commitment on the part of the researcher that has the potential to facilitate further extended access to all participants. When staff and students see the researcher investing in a project, it can encourage them to support and participate in a researcher's studies. A positive by-product of the reciprocal approach is that researchers spend more time with the students and staff, getting to know their individual behavioral traits thus becoming more attuned for the design and evaluation process. Negotiating these combined roles of researcher, TA, workshop leader, technical facilitator is a necessary part of the research process when being embedded within a school. However, taking on these combined roles has both a positive and detrimental effect on the ability of the researcher to conduct research to the best of their ability.

\section{REFLECTIONS}

The rapidly fluctuating dynamic of the SEN school environment is one that can take some time with which to become acclimatised. For example, the second author has had difficulty in securing space and equipment when attempting to conduct studies. Additionally, as the classroom context is no different, the researcher has also run into difficulty where students have physically manipulated the recording equipment rendering the recordings of the sessions unusable for analysis. This is a point that will need to be considered when designing any studies in the special school context.

The issue of communication is clearly one of the most important points for consideration when conducting design research in the SEN classroom setting. The first two authors have found it difficult to maintain this communication throughout the various phases of their research projects, resulting in some avoidable detrimental effects. When there is a lack of communication between those involved in the study, it makes it difficult for the staff members in the school to factor the research into their general timetables. As an example, the second author has travelled to the school without confirmation on more than one occasion to find that a particular participant is not present that day, or that the class teacher is away and so the schedule has changed. It can also prove to be an obstacle for maintaining good relationships within the institution, particularly if perceived obligations are not met by the researcher. It has become apparent however, that when the researchers have presented their plans in the most succinct manner possible, staff members and parents of students have been very willing to allow the students to participate in the studies.

The first two authors have found it a distinct struggle to understand how to elicit useful information from earlyyears, non-verbal design partners and subsequently how this information can be translated into design outcomes. The ECHOES [4] project details the technique of mindful interpretation while undertaking these sorts of practices however they still feel like there is a lack of clarity in the literature as to make strong connections between workshop products and design guidelines.

The practice of showing commitment by positive contributions and regular attendance has also proven to be a very useful tool when working in the SEN context. During the periods when the researchers have been able 
to do this, it has resulted in increased cooperation and help from the staff in the school. Similarly, throughout the periods of undertaking the extra contributions, the researchers have observed staff becoming more at ease with their presence. The positive outcome of this is that more fine grained-detail regarding students behavior and responses can be gleaned as members of staff communicate in a less formal way. For example, a student may not respond well to an intervention for the first two short sessions, but this reaction could change substantially when implemented over the course of a term. Both researchers have had to undertake a period of renegotiation after a phase in the project that has meant that they cannot be present in the school on a regular basis.

During their studies the researchers have made use of their technical and artistic skills to support and lead arts based activities in the classroom. They have worked with creative technologies already present in the school as well as designing and building new ones. This has included a term length digital story telling project with three classes resulting in a set of public performances. It has helped them to build a strong relationship with staff and students and creates a space to observe how participants work with creative technologies in the classroom.

Working as PhD candidates the researchers often work alone and with a limited amount of time and resources. When their position as a researcher works at crosspurposes with many other roles undertaken, the aims of the study and the relationships developed with the school, its staff, and students can become compromised. The responsibilities taken on as researchers embedded in a special needs school are necessarily challenging but in both the first two authors experience, they have found points where they have been overwhelmed by the sheer volume of work these roles engender. How these roles are negotiated to fit within the agendas of the research institution and the school is something which both of the first two authors are learning is difficult but essential when becoming embedded in a classroom context.

\section{CONCLUSION}

The key factors regarding conducting embedded research in a SEN school in the UK have been presented. These include awareness of the various institutions and people within them that are involved, the dynamic of the school environment and managing relationships whilst conducting the studies. The importance of maintaining good relationships has been highlighted because the staff in the school are so integral to conducting a successful study with limited resources. By ensuring regular and succinct communication, a willingness to commit to extra activities and managing the various roles they take, it is possible for researchers to carry out their studies in this fluctuating and unpredictable environment. Although pursuing an embedded approach as detailed above is a complex and at times demanding approach to research in this field, it has been exciting, enjoyable and highly rewarding. Through their studies the researchers have developed strong and lasting relationships that have opened a rich space for the effective and sustained development of interactive technologies in SEN settings.

The experiences detailed thus far have provided insight into some of the main points for consideration when conducting research in this field. While they have not always been successful in navigating these potential pit falls, other researchers and practitioners can be aware of them when undertaking their own studies in this diverse and constantly surprising context. The points to consider further now are:

- How to leave a positive design legacy with the research partners once the initial scope of the project has ended?

- Is there a boundary to which participants' communication abilities for example, young people with profound and multiple learning difficulties, prevent them from being design partners? What techniques can be employed to involve these groups in the design process?

\section{ACKNOWLEDGMENTS}

The authors would like to emphasise the importance of the support and commitment of staff and students in Three Ways School.

\section{REFERENCES}

[1] P. H. Christensen. Children's participation in ethnographic research: Issues of power and representation. Children \& Society, 18(2):165-176, Apr. 2004.

[2] A. Druin. The role of children in the design of new technology. Behaviour and Information Technology, 21(1):1-25, 2002.

[3] T. Falcã o and S. Price. Informing design for tangible interaction: a case for children with learning difficulties. IDC '10: Proceedings of the 9th International Conference on Interaction Design and Children, June 2010.

[4] C. Frauenberger, J. Good, and W. Keay-Bright. Designing technology for children with special needs: bridging perspectives through participatory design. CoDesign, 7(1):1-28, Mar. 2011.

[5] J. Good and J. Robertson. Carss: A framework for learner-centred design with children. International Journal of Artificial Intelligence in Education, 16(4):381413, 2006.

[6] M. Guha and A. Druin. Designing with and for children with special needs: an inclusionary model on Interaction design and children, 2008.

[7] A. Hornof. Working with Children with Severe Motor Impairments as Design Partners. In Proceedings Interaction Design and CHildren IDC '08, pages 69-72, Apr. 2008.

[8] E. Ka rna , J. Nuutinen, K. Pihlainen-Bednarik, and $\mathrm{V}$. Vellonen. Designing technologies with children with special needs: Children in the Centre (CiC) framework. IDC '10: Proceedings of the 9th International Conference on Interaction Design and Children, June 2010.

[9] J. McElligott and L. Leeuwen. Designing sound tools and toys for blind and visually impaired children. IDC '04: Proceedings of the 2004 conference on Interaction design and children: building a community, June 2004.

[10] S. L. Odom, E. Brantlinger, R. Gersten, R. Horner, B. Thompson, and K. Harris. Research in special education: scientific methods and evidence-based practices. Exceptional Children, 71(2):137, 2005. 